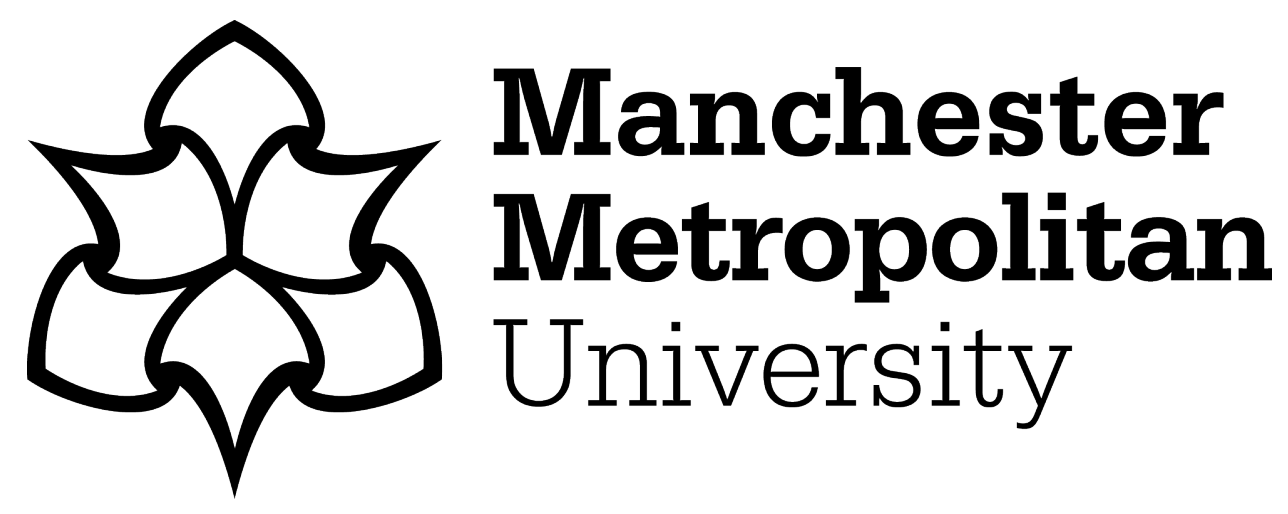

Rehman, F, Masood, H, Ul-Hasan, A, Nawaz, Raheel ORCID logoORCID: https://orcid.org/0000-0001-9588-0052 and Shafait, F (2019) An Intelligent Context Aware Recommender System for Real-Estate. In: 3rd Mediterranean Conference on Pattern Recognition and Artificial Intelligence, MedPrai 2019, 22 December 2019 - 23 December 2019, Istanbul, Turkey.

Downloaded from: https://e-space.mmu.ac.uk/625002/

Version: Accepted Version

Publisher: Springer

DOI: https://doi.org/10.1007/978-3-030-37548-5_14

Please cite the published version 


\title{
An Intelligent Context Aware Recommender System For Real-Estate
}

\author{
Faiza Rehman ${ }^{1}$, Hira Masood ${ }^{1}$, Adnan Ul-Hasan ${ }^{2}$, Raheel Nawaz $^{3}$, and \\ Faisal Shafait ${ }^{1,2}$ \\ 1 School of Electrical Engineering and Computer Science, \\ National University of Sciences and Technology (NUST), Islamabad, Pakistan \\ $\{$ faisal.shafait\}@seecs.edu.pk \\ 2 Deep Learning Lab, National Center for Artificial Intelligence, Islamabad, Pakistan \\ 3 School of Computing, Mathematics and Digital Technology, Manchester \\ Metropolitan University, Manchester, U.K. \\ R. Nawaz@mmu.ac.uk
}

\begin{abstract}
Finding products and items in large online space that meet the user needs is difficult. Users may spend a considerable amount of time before finding item relevant to their needs. Like many other economic branches, growing Internet usage also change user behavior in the real-estate market. Advancement in virtual reality offers a virtual tour, interactive maps, floor plans that make an online rental website popular among users. With an abundance of information, recommender systems become more than ever important to suggest the user with relevant property and reduce search time. A sophisticated recommender in this domain can assist the need of a real-estate agent. Session-based user behavior, lack of user profile leads to the use of traditional recommendation methods. In this research, we proposed an approach for real-estate recommendation based on Gated Orthogonal Recurrent Unit (GORU) and Weighted Cosine Similarity. GORU captures the user searching context and weighted cosine similarity improves the rank of pertinent property. To conduct this research, we have used the data of an online public real estate web portal ${ }^{4}$. The factual data represents the original behavior of the user on an online portal. We have used Recall, User coverage and Mean Reciprocal Rank (MRR) metric for the evaluation of our system against other state-of-the-art techniques. Proposed solution outperforms various baselines and state-of-the-art RNN based solutions.
\end{abstract}

Keywords: Recommender Systems · Deep learning · Real Estate · GORU - RNN.

\section{Introduction}

The recommender system is a class of information retrieval domain. The main purpose of the recommendation system is to improve the consumer experience

\footnotetext{
4 AARZ.PK
} 
and provide with the items that are relevant to users. Recommender system is a software utility that provides suggestions for the item to be used by the user [17]. Relying on these suggestions, users make various decisions including which movie to watch, which song to hear, which news to read, which house to purchase, etc. These systems have proved to be helpful when a user deals with an overwhelming amount of information searching online through pervasive large item space. The online product catalogs evolve continuously to include highvalue products such as apartments and computers; hence, the task of locating the desired choice among a large set of options is intimidating for an average customer [3].

Like many other fields, the internet has also reshaped the behavior of a consumer and a supplier in the real estate domain. It is very difficult for both the supplier and customer to survey the real estate market physically, whereas an online real-estate portal provides an abundance of information with just a few clicks. Advancements in virtual reality provide the facility of a virtual tour. Introduction of an interactive map and floor plan makes online real estate portal user's first choice for property search. By integrating sophisticated recommender system, online search time can be reduced and the need for a real-estate agent in property sell and purchase can be assisted. Real estate market tends to have a big impact on the economy of any country. In US, the real estate industry accounted for $\$ 3,372,634$ million or $17.3 \%$ of the gross state product in 2017 .

Despite the importance of a recommender system in real estate domain, it has been a relatively unappreciated problem in research community. Most of the literature in our research comes from the generic recommender system techniques. Mostly research in recommender system focused on the models where users proper identifiers and profiles are available. In this setting, the matrix factorization based approaches and neighborhood model dominated the literature [7]. Whereas in the real system the website rarely saves users identifier. Even if tracking is possible, the user rarely visit the rental portal for same purpose; instead they usually exhibit session based behavior. Lack of user profile with session based behavior leads to the use of relatively simple methods that do not take into account the user profile, i.e., item to item similarity. These methods only take into account last click of the user ignoring the context of user search.

In this work, we proposed a deep learning based recommender system particularly for real estate domain for efficient online searching.Proposed approach recommends properties based upon user searching context rather than just last clicked item. Section 2 overviews the related work on recommender system. Section 3 describes the details of our methodology. Section 4 outlines the experimental details including the features used, experimental setups, results, and comparison with the baseline methods. Section 5 concludes the paper and provide points for future research. 


\section{Related Work}

Despite many years of research, the recommender systems in real-estate has remained less focal area in the research sphere. One of the first approach that highlights the application of a recommender system in real estate was proposed by Shearin et al. [18]. The proposed system attempted to reproduce suggestion similar to a human real estate agent. The system based upon an interactive learning procedure which was not further particularize in this study. It was based upon extensive user feedback, to gather user view about various aspect of attributes of properties. The information gathered through questioners used to set the standard filter on the database and make a recommendation about relevant properties.

Graaff et al. [5] proposed a geosocial recommender system that used data from different data sources. The system was capable to make recommendations about local businesses. It mainly used data from social media. The proposed approach applied profile matching mechanism. The profile matching based upon the collaborative filtering to make suggestions. The authors claimed that concept of geoprofile can be shift to real estate domain. However, this approach was dependent on social media data to make suggestions.

Yuan et al. [22] proposed an approach to improve the efficiency and affordability of an online housing search. They proposed a user-oriented recommender system for real-estate portal using ontology and case-based reasoning relied upon the user search behavior. They aimed to find the semantic construction of housing unit information and designed all the sub-modules for building a recommender system. They used questionnaire and user search behavior for semantic relationship construction. The extracted ontology contained information about the knowledge collected in the user study and real estate domains. Finally uses case representation and case indexes to find the similarity between search queries and user cases.

A simulation-based recommender study using real estate data was published by Chulyadyo et al. [12]. They proposed a personalized Probabilistic Relational Model (PRM) based on users preferences in decision making. They also demonstrated that the same PRM can be used to achieve content-based, collaborative filtering and hybrid approaches.

The first ever machine learning based approach in real-estate recommender system was proposed by Julian et al. [14]. In their research, they used MLP to make recommendations. The proposed solution makes recommendations based upon rating given by the user to a specific property. The MLP takes user id and property features as input and predicts a rating for the property that the user has not already rated. They also showed the various possibility of embedding recommender in the real-estate portal during the user search journey. The algorithm required explicit feedback from the user to make it work. And for every recommendation, the algorithm has to predict rating for all the property available in the system. 


\subsection{Recurrent Neural Network for Recommendation}

RNN is a deep model that works particularly well when dealing with sequence or temporal data. RNNs have been successfully used in image and video captioning, time series prediction, natural language processing. Long short-term memory (LSTM) [11] are a type of RNN that works particularly well. It includes additional gates that control the hidden state of RNN. This helps in vanishing gradient problem that seems to raise in standard RNN.

Gated Recurrent Unit (GRU) [4] is comparatively simpler then LSTM. Recently URNN and GORU have been proposed that helps in reducing gradient explosion problem. In this work, we have used GORU [13] for real-estate recommendation problem. GORU combines the remembering ability of unitary RNN to remember the long-term dependencies and gated RNN to forget the irrelevant information.

RNNs were first used to model session data in [9]. This GRU based recurrent neural network is trained with the pairwise ranking loss function Top1.This network takes clicked item IDs as input and provide recommendation after each click. Parallel RNNs were also used to incorporate item features, i.e., textual description and a thumbnail image of the item to make recommendations [10]. This technique provides a better recommendation as compared to plain RNNs but has the drawback of increased training time.

In [19] the author used data augmentation technique to improve the performance of RNN in session-based recommendation. This technique has the drawback of increased training time as in this work single session has been split into sub-sessions. RNNs have also been used in user-item collaborative filtering [21] [6] where results are not up to the par and hardly outperforms matrix factorization method. In [16] authors proposed the hierarchical RNN (HRNN) to make recommendation in the scenario when information about the users previous session is available.

In [15] the authors proposed a recommender system for Carpooling services which allows the drivers to share rides with other passengers. This reduces the passengers' fares and time, as well as traffic congestion and increases the income for drivers. Another recommender system for recommending movies was proposed in [2] which used the content based filtering approach with fuzzy logic and conformal prediction algorithm.

The goal of our study is to use novel sequence model GORU and URNN [1] for recommendation. Results show that GORU based model train faster with less number of epochs and hidden units. At the same time, it provides performs better in terms of different measures as compared to GRU and URNN. It also outperforms other state of the art session based recommendation technique with a significant margin.

\section{$3 \quad$ Methodology}

Our proposed system consist of two main modules. First is candidate generation module which is based upon deep learning a deep learning (i.e. Gated 


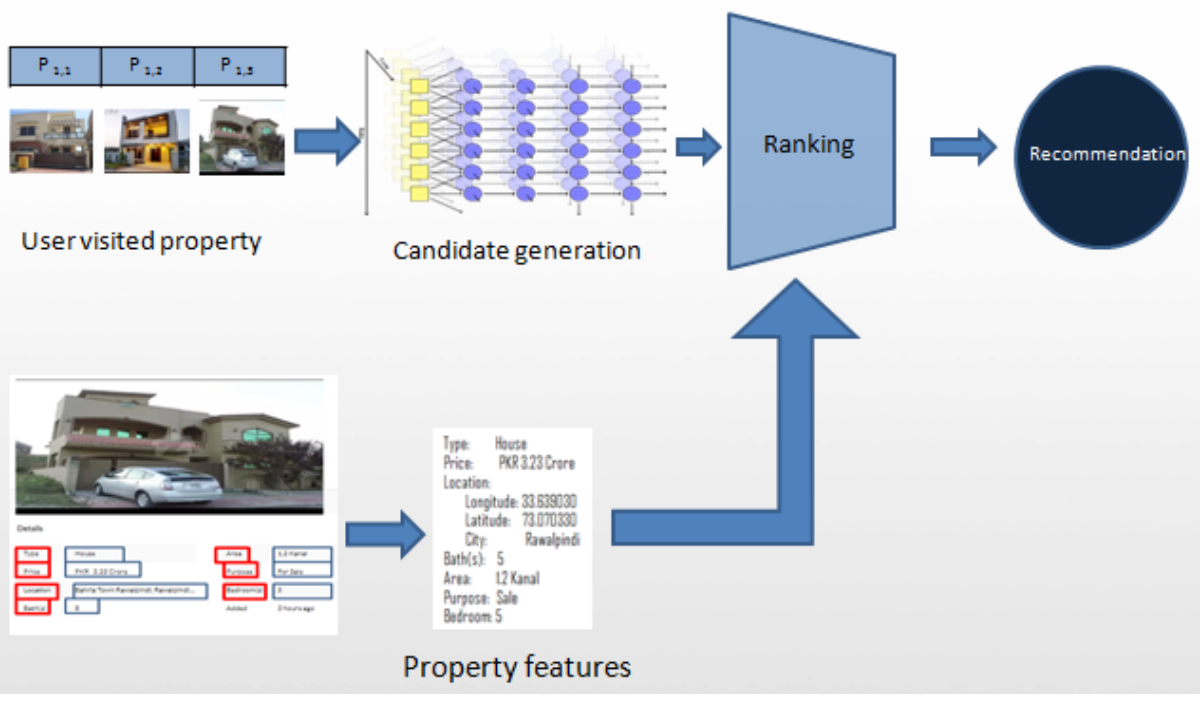

Fig. 1. An illustration of the proposed approach, Candidate generation module based upon deep learning takes one hot-encoding of property-ID and generate candidate properties, Ranking module takes candidate properties and apply Weighted Cosine Similarity between last click property and candidate properties.

Orthogonal Recurrent Unit) and the second is the ranking module. Candidate generation module generates properties based upon the user searching context. Ranking module takes candidates properties as input and by using weighted cosine similarity improves the rank of relevant property. Figure 1 depicts the proposed solution.

\subsection{Candidate Generation using GORU}

This module is based on the RNN-based Gated Orthogonal Recurrent Unit (GORU). GORU models user's searching behavior on the online real estate portal. For our study we use data of a online real-estate portal. Our model takes 1 of $\mathrm{N}$ representation of user searched property ID and output the probability of next possible items.

In our setup, we use a ranking loss function TOP1. For training, we use sequences of user clicked property IDs generated in session during searching online real estate website and model user searching behavior. This module serves as the unit to generate the property IDs that are most likely to be the user relevant property. Properties with a high probability serve as the candidates. Figure 2 shows the process of candidate generation. 


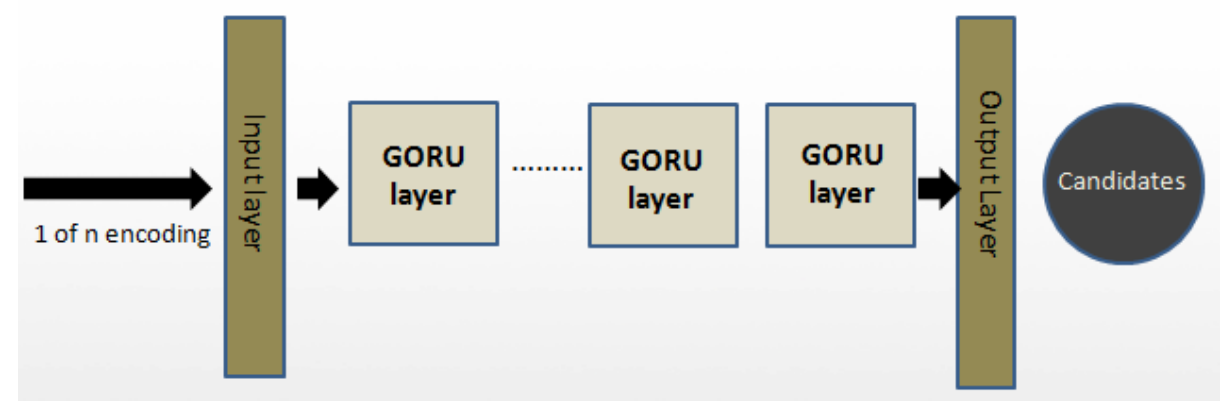

Fig. 2. Candidate Generation using GORU, Input layer takes 1 of $\mathrm{N}$ encoding of visited property ID and pass it to GORU layers, Output layer gives the probability corresponding to all the properties

Gated Orthogonal Recurrent Unit Recurrent neural network has devised to model sequential data. The hidden state $h_{t}$ of RNN makes them different from the feed-forward neural network.

$$
h_{t}=g\left(W x_{t}+U h_{t-1}\right)
$$

where $g$ is the activation function. $x_{t}$ is the input at time step $t, h_{t-1}$ is previous hidden state.

GRU is gated model of RNN that deals with the vanishing gradient problem. Gates controls the changes of the hidden state The hidden state in GRU is the linear interpolation of previously hidden state and candidate hidden state.

$$
h_{t}=\left(1-z_{t}\right) h_{t-1}+z_{t} \hat{h}_{t}
$$

where $z$ is the update gate computed as

$$
z_{t}=\sigma\left(W_{z} x_{t}+U_{z} h_{t-1}\right)
$$

while the candidate hidden state $\hat{h_{t}}$ is computed in a similar manner

$$
\hat{h_{t}}=\tanh \left(W x_{t}+U\left(r_{t} \odot h_{t-1}\right)\right.
$$

and finally, the reset gate $r t$ is given by

$$
r_{t}=\sigma\left(W_{r} x_{t}+U_{r} h_{t-1}\right)
$$

A complex-valued matrix $O$ is unitary when it satisfies $O O^{T}=I$ where I is the identity matrix. and real-valued unitary matrix is called orthogonal. Any vector multiplied by orthogonal matrix satisfies this. A complex-valued matrix $\mathrm{U}$ is unitary when it satisfies $O O^{T}=I$.

$$
\|O x\|=\|x\|
$$




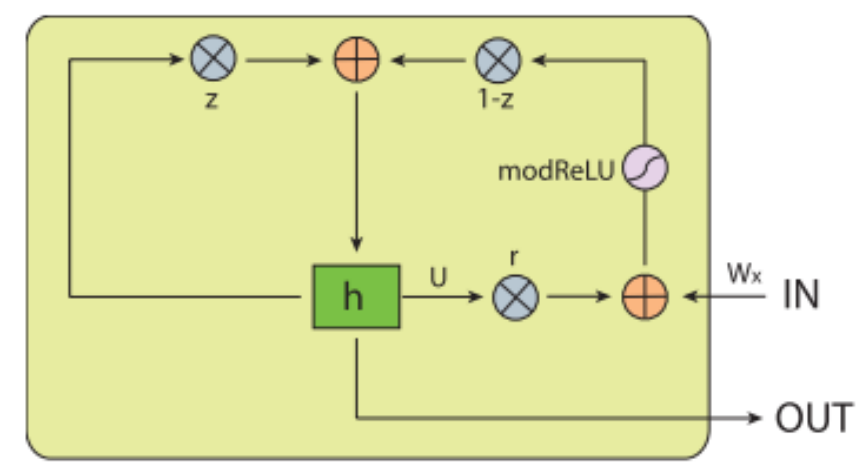

Fig. 3. An illustration of GORU [13], $h$ is the hidden state. $z$ and $r$ are update and reset gate, here $U$ is the orthogonal matrix.here activation function in $\bmod R E L U$

Due to above property the $\mathrm{U}$ matrix is able to preserve the norm of matrix passing through it and allow the gradient to propagate longer time. In our scenario, to use GORU we change the hidden state matrix in orthogonal matrix and activation function to modRELU given as

$$
\begin{gathered}
h_{t}=z_{t} \odot h_{t-1}+\left(1-z_{t}\right) \odot \hat{h_{t}} \\
\hat{h_{t}}=\bmod \operatorname{ReLU}\left(W_{x} x_{t}+r_{t} \odot\left(O h_{t-1}\right)+b_{h}\right)
\end{gathered}
$$

where $\hat{h_{t}}$ is candidate hidden state.

Figure 3 represents the working of GORU. We use 1-of-n encoding with session parallel mini-batches. We also tried other sequence learning algorithms i.e. GRU, URNN and LSTM. Results show that GORU outperforms others. We also tried different loss function, TOP1 and BPR show competitive results and outperforms other.

1 of $\mathbf{N}$ Encoding The input of the network is the current state of the session while the output is the item of the next event in the session. For input, we use 1 of $\mathrm{N}$ encoding representation of the item. The length of the input vector equals the number of items and only the coordinate corresponding to the current item is one, the others are zeros. We also experimented with adding an additional embedding layer, but the 1-of-N encoding always outperforms.

Session Parallel Mini Batches Mostly RNNs use in-sequence mini-batches for natural language processing tasks. For example, it is common practice to use a sliding window over the sentences and put these windowed segments adjoining to each other to form mini-batches. This strategy does not suit our task, because (1) The length of sessions can be very diverse, even more than that of sentences: 


\section{Session1 $14,1\left|\frac{1,2}{i_{1,3}}\right|_{1,4}$}

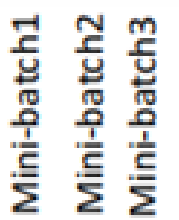

Session2 $2,1 k_{2,2} k_{2,3}$

Session3 3 \begin{tabular}{l|l|l|l|l|l|}
\hline 3,1 & $i_{3,2}$ & $i_{3,3}$ & $i_{3,4}$ & $i_{3,5}$ & $i_{3,6}$ \\
\hline
\end{tabular}

Session4 $4,1 / 4,2$

Session5 \begin{tabular}{|l|l|l|}
\hline 5,1 & $k_{5,2}$ & $k_{5,3}$ \\
\hline
\end{tabular}

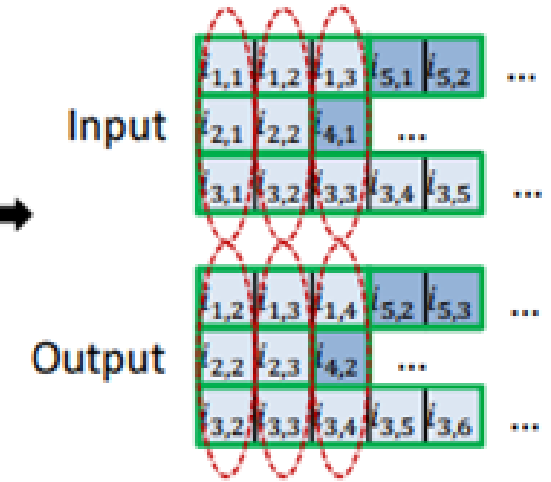

Fig. 4. Session Parallel Mini Batches

some sessions consist of only 2 events, while others may range over a few hundreds of events. (2) Our goal is to capture how a session progress over time, so breaking down into segments would make no sense.

For that, we use session-parallel mini-batches depicted in Figure 4 . Firstly, we create an order for the sessions Then, we put the first occurrence of the first $\mathrm{X}$ sessions to form the input of the first mini-batch (the required output is the second occurrence of our active sessions). The second mini-batch consist of the second occurrence and so on. If any of the sessions terminate than the next available session is put in its place. Sessions are considered independent, thus we reset the suitable hidden state when this session switch occurs.

Ranking Loss Function The key to recommender systems is the relevancebased ranking of recommended items. Although this task can also be considered as a classification task but learning-to-rank approaches generally outperform other approaches. Ranking can be point-wise, pairwise and list-wise.

Point-wise ranking independently estimates the score or the rank of items, and the loss is defined in such a way so that the rank of relevant items should be low. The pairwise ranking compares the score or the rank of pairs of a positive and a negative item and the loss impose that the rank of the relevant or positive item should be lower than that of the negative item. List-wise ranking uses the scores and ranks of all items and compares them to the perfect ordering. As this one requires sorting so it is computationally expansive and not used of- 
ten. In our case, we use pair-wise loss function named TOP1 proposed by Hidasi et.al [9] specifically for recommendation task. It is the regularized approximation of the relative rank of the pertinent property. The relative rank of the pertinent property is given by $L_{s}=\frac{1}{N_{S}} \cdot \sum_{j=1}^{N_{S}} I\left\{\hat{r}_{s, j}>\hat{r}_{s, i}\right\} . I\{$.$\} is approximate with a$ sigmoid function. Optimizing for this would modify parameters such that the score for i would be high. But in some cases, certain relevant items also act as a negative example and score tends to become increasingly higher. To circumvent this, we want to impose the scores of the negative sample to be near zero. Which is the organic expectation towards the scores of negative items, Thus regularization term has added to the function. This regularization term must be in the same range as the relative rank and acts similarly to it. Finally, the loss function is as follows: $L_{s}=\frac{1}{N_{S}} \cdot \sum_{j=1}^{N_{S}} \sigma\left\{\hat{r}_{s, j}>\hat{r}_{s, i}\right\}+\sigma r^{2}{ }_{s, j}$

\subsection{Ranking}

In this module, we took generated candidates as input and measure the similarity of all candidate to users last clicked item. Similarity measure we use in our study is weighed cosine similarity. According to Tonara et al. [20] survey, they identify 7 major deciding factors in real estate sales and purchase. These criteria are price, number of room, number of bathrooms, location, building area, land area, and house certificate. As in online website, the information about the house certificate is not available so we exclude this and we identify two further deciding factors, Property type (i.e. house, plot, flat etc) and property purpose (i.e. whether it is available for rent or sale). And the location is subdivided in City, longitude, and latitude. Each property is represented as the array of attributes. We used min-max normalization for features normalization.

$$
\begin{gathered}
P 1=\text { (PropertyType, PropertyPurpose, City, Longitude, } \\
\text { Latitude, Area, NumRooms, NumBaths })
\end{gathered}
$$

Assign weights according to the relative importance of features.

$$
\begin{gathered}
P 1=\left(w_{1} \times \text { Property Type, } w_{2} \times \text { PropertyPurpose, } w_{3} \times \text { City, } w_{4} \times \text { Longitude },\right. \\
\left.w_{5} \times \text { Latitude, } w_{6} \times \text { Area, } w_{7} \times \text { NumRooms, } w_{8} \times \text { NumBaths }\right)
\end{gathered}
$$

For some candidate property C:

$$
\begin{gathered}
C 1 \text { = (PropertyType, PropertyPurpose, City, Longitude, } \\
\text { Latitude, Area, NumRooms, NumBaths) }
\end{gathered}
$$

Assign weights according to the relative importance of features.

$$
\begin{gathered}
C 1=\left(w_{1} \times \text { PropertyType, } w_{2} \times \text { PropertyPurpose, } w_{3} \times \text { City, } w_{4} \times\right. \text { Longitude } \\
\left.w_{5} \times \text { Latitude, } w_{6} \times \text { Area, } w_{7} \times \text { NumRooms, } w_{8} \times \text { NumBaths }\right)
\end{gathered}
$$




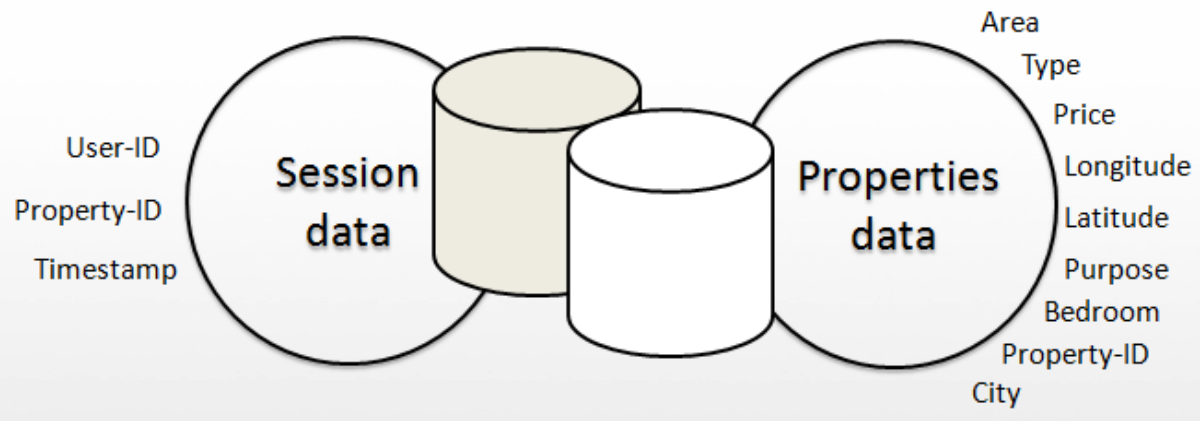

Fig. 5. Session data contains information about user behavior in session. Property data contain information about the particular real estate property.

$$
\cos (\mathbf{P} \mathbf{1}, \mathbf{C} \mathbf{1})=\frac{\mathbf{P} \mathbf{1} \mathbf{1} \mathbf{1}}{\|\mathbf{P} \mathbf{1}\|\|\mathbf{C} \mathbf{1}\|}=\frac{\sum_{i=1}^{n} \mathbf{P} \mathbf{1}_{i} \mathbf{C} \mathbf{1}_{i}}{\sqrt{\sum_{i=1}^{n}\left(\mathbf{P} \mathbf{1}_{i}\right)^{2}} \sqrt{\sum_{i=1}^{n}\left(\mathbf{C} \mathbf{1}_{i}\right)^{2}}}
$$

The cosine similarity measure is used to find the similarity between generated candidates and last clicked property. Candidate properties with high similarity value use as recommendations. The result shows that this module improves the ranking of the relevant property.

\section{Experimental Evaluation}

To thoroughly evaluate our algorithm, we have performed a number of experiments. This section describes the dataset used and the protocol followed, besides outlining the key results obtained.

\subsection{Data Description}

We have obtained data of an online real-estate portal. It consists of two types of data, Google analytic data of the online portal that contain session-based user data, user browsing history, (this session based data grabs the user behavior in the form of click stream) and the other is the property attributes stored in the database of the online portal.

Figure 5 shows the data we have used in our research study. The session based data demonstrate the user's session based behavior. Property data contains information about property attributes that the users are concerned about while purchasing property. These include primary attributes such as the number of rooms, area, and the location of the property, as well as derived attributes such as distance to school, distance to shopping center, etc. These attributes are fundamental to establishing similarity between properties and identifying a particular user's interest.

The following table (Table 1) shows session data that we have used for model training and testing purpose. 
Table 1. The specification of the data set used in our study

\begin{tabular}{|c|r|r|}
\hline Data & Number of Events & Number of Session \\
\hline Train Data & 26,121 & 6,390 \\
Test Data & 8,238 & 2,608 \\
\hline
\end{tabular}

\subsection{Baselines}

The following are the approaches mainly used in session-based recommendation systems.

POP: Popularity predictor that always recommends the most popular item. Although this approach is simpler, but widely used in practical system and in certain domain it acts as a strong baseline.

S-POP: Recommend most popular item in the current session. In our setting, we choose most popular item in the current batch.

KNN: It is item to item collaborative filtering approach in which item similar to the current items is recommended. Cosine similarity measure is used to find the similarity between the items session vectors.

BPR-MF: BPR-MF is a matrix factorization method. To make it work in a session based setting, we used the same approach as described in [9].

GRU4rec:. It is the first system that applies RNN in recommender system [9]. In this system, GRU has been used to generate the recommendations. This paper also serves as the basis for our candidate generation module as well.

RNN with TOP-K Gain: This applies RNN with a new rank function [8]. They introduced a new class of loss functions that together with an improved sampling strategy provide top-k gains for RNNs.

\subsection{Evaluation Metrics}

Recall: Recall is also known as the sensitivity. it is the fraction of relevant item retrieved through recommender over the total number of relevant item.

$$
\text { Recall }=\frac{\text { relevant_items } \cap \text { recommended_items }}{\text { relevant_items }}
$$

MRR: In information retrieval and recommender system the rank of the relevant item is also important, i.e., at which rank the system is retrieving the relevant item. Mean reciprocal rank (MRR) is the average of inverse of rank given to the first relevant item.

$$
M R R=\frac{1}{S} \sum_{i=1}^{S} \frac{1}{\operatorname{rank}_{i}}
$$

Here $\operatorname{rank}_{i}$ is the rank given to the first recommended relevant item. $S$ is the number of sessions. 
User _ coverage: User coverage is the fraction of number of users getting correct recommendation over total number of users getting recommendation.

$$
\text { User_coverage }=\frac{U_{r}}{U}
$$

Here $U_{r}$ is the number of user get relevant recommendations. $U$ is the total number of users. In our scenario the number of users is the same as the number of sessions.

\subsection{Results and Discussion}

We optimized the hyperparameter by running multiple experiments and randomly selecting parameter points. Then the parameters was further optimized by tuning each parameter separately. The optimization was done on a separate validation set, then evaluated on test data. We have experimented with different sequence model i.e. LSTM, GRU, URNN, and GORU. For our experiments, we have used GeForce Titan X GPU. However, even on CPU it can be trained in a acceptable time.

Figure 6(a) shows the loss vs epoch graph for different sequence model. Note that GORU shows faster convergence than other sequence models, besides achieving the lowest loss after 20 epochs. The loss function of GRU showed a steady decline but the convergance was slow. Therefore, we chose GORU as the model of choice in our study. Figure 6(b) shows the effect of using different learning rates when training GORU. According to the results, a learning rate of 0.01 performs the best. It is interesting to note that both high learning rate of 0.1 and low learning rate of 0.0001 resulted in poor convergance. We have also tried different optimizers i.e. RMSprop, Adagrad, Adadelta and Adam. Figure 6(c) shows the convergence for different optimizer. In our case Adam performed better than others, while AdaDelta could not converge. We have experimented with different loss function i.e. TOP1, BPR, TOP1-MAX, CCE, Blackout. In terms of evaluation metric TOP1 and BPR both gives competitive results. Blackout perform worst but at the same time increase diversity factor.

Our proposed Hybrid GORU approach outperforms various baselines including RNN based solutions as well. Table 2 shows the result of the best performing network. Error analysis shows that this network performs particularly well for long sessions and the items that have higher support count i.e. the properties that are mostly visited. GORU based approach has significant gain over previous approaches included GRU based as well. By increasing the number of hidden units within a single layer, results can be further improved with respect to all the evaluation metrics whereas adding layers decreases the performance of the network. In terms of the loss function, BPR and TOP1 both give competitive results and outperforming other loss function i.e. CCE and Blackout [6].

The proposed approach is popularity based hence it doesn't work well for rarely visited items. Moreover, the database of real estate portal tends to update frequently. This change requires the retraining of model frequently to integrate new properties in the recommendation. 


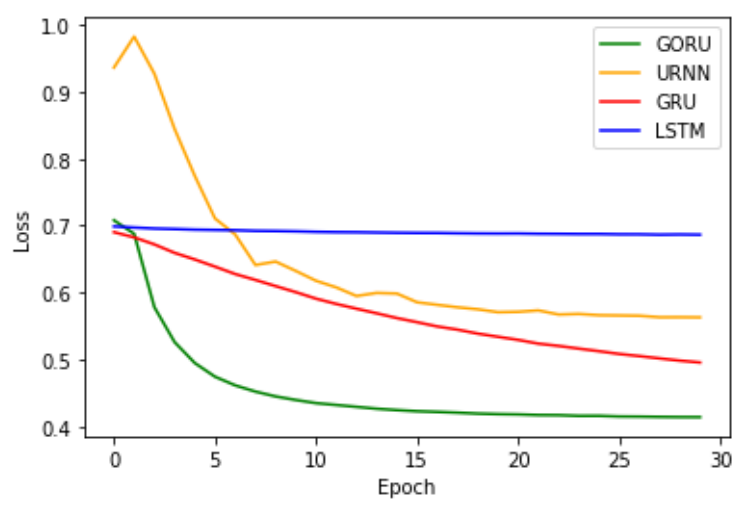

(a) A comparison of different sequence models

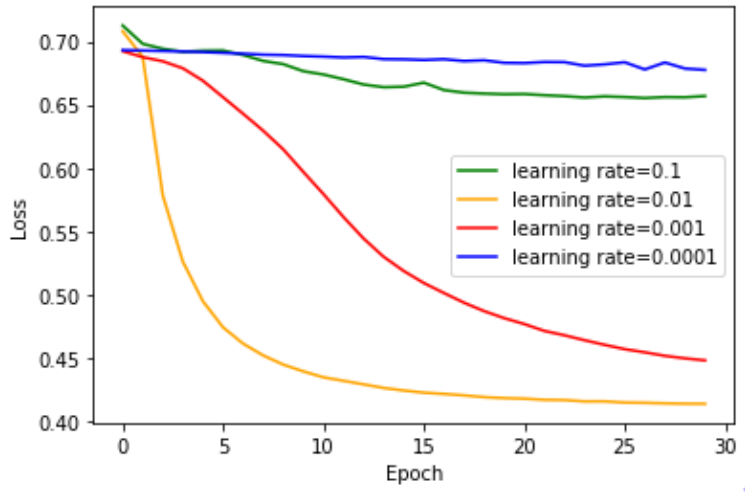

(b) A comparison of different learning rates

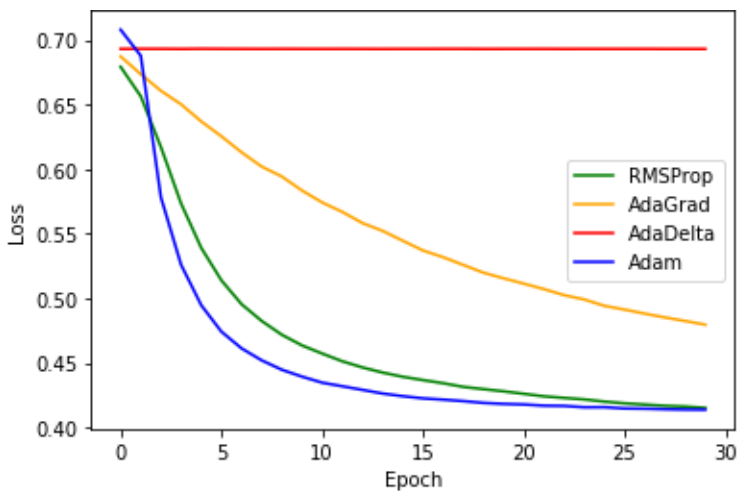

(c) A comparison of different optimizers

Fig. 6. Loss vs Epoch graphs for different parameters 
Table 2. A comparison of the proposed method with different baseline methods. The results show a clear superiority of the presented method as it significantly outperforms the baseline methods on all evaluation metrics.

\begin{tabular}{lrrr}
\hline Method & User- Coverage Recall @20 MRR@ 20 \\
\hline & & & \\
POP & 0.9 & 0.7 & 0.1 \\
S-POP & 31.6 & 35.8 & 26.2 \\
KNN & 40.9 & 35.4 & 14.2 \\
BPR-MF & 29.4 & 32.0 & 25.2 \\
GRU4rec [9] & 34.6 & 30.9 & 17.3 \\
TOP-K Gain [8] & 43.1 & 37.5 & 19.1 \\
Hybrid GORU & $\mathbf{5 8 . 5}$ & $\mathbf{5 9 . 9}$ & $\mathbf{2 9 . 3}$ \\
\hline
\end{tabular}

\section{Conclusion}

The personalized real estate recommender system can help the user to find out the relevant property in less searching time. Although our ranking module requires manually static weights however it can be used to incorporate the business needs as well e.g. increase diversity in the recommendation, give priority to the candidate property whose advertisement has submitted earlier than others. Our work can serve as a basis to explore the real estate domain further with deep learning techniques.

Advance features in online real-estate portal i.e. virtual tour interactive maps $3 \mathrm{~d}$ tours etc. attracts increasingly large visitors. To accommodate a large number of online visitor, implementation of this recommender system for the distributed platform would be an effort worth making both for its academic value and market potential.

\section{Acknowledgment}

This research was partly supported by HEC Grant TDF-029.

\section{References}

1. Arjovsky, M., Shah, A., Bengio, Y.: Unitary evolution recurrent neural networks. In: International Conference on Machine Learning. pp. 1120-1128 (2016)

2. Ayyaz, S., Qamar, U., Nawaz, R.: Hcf-crs: A hybrid content based fuzzy conformal recommender system for providing recommendations with confidence. PLOS ONE 13(10), 1-30 (10 2018). https://doi.org/10.1371/journal.pone.0204849

3. Chen, L., Pu, P.: Preference-based organization interfaces: Aiding user critiques in recommender systems. In: User Modeling (2007)

4. Chung, J., Gulcehre, C., Cho, K., Bengio, Y.: Empirical evaluation of gated recurrent neural networks on sequence modeling. arXiv preprint arXiv:1412.3555 (2014) 
5. De Graaff, V., van Keulen, M., de By, R.A.: Towards geosocial recommender systems. In: Proceedings of the 4th International Workshop on Web Intelligence \& Communities. p. 8. ACM (2012)

6. Devooght, R., Bersini, H.: Collaborative filtering with recurrent neural networks. CoRR abs/1608.07400 (2016)

7. F. Anwaar, N. Iltaf, H.A.R.N.: Hrs-ce: A hybrid framework to integrate content embeddings in recommender systems for cold start items. Journal of Computational Science 29, 9 - 18 (2018)

8. Hidasi, B., Karatzoglou, A.: Recurrent neural networks with top-k gains for sessionbased recommendations. In: CIKM (2018)

9. Hidasi, B., Karatzoglou, A., Baltrunas, L., Tikk, D.: Session-based recommendations with recurrent neural networks. arXiv preprint arXiv:1511.06939 (2015)

10. Hidasi, B., Quadrana, M., Karatzoglou, A., Tikk, D.: Parallel recurrent neural network architectures for feature-rich session-based recommendations. In: Proceedings of the 10th ACM Conference on Recommender Systems. pp. 241-248. ACM (2016)

11. Hochreiter, S., Schmidhuber, J.: Long short-term memory. Neural computation 9(8), 1735-1780 (1997)

12. Hodoň, M., Eichler, G., Erfurth, C., Fahrnberger, G.: Innovations for Community Services. Springer (2018)

13. Jing, L., Gulcehre, C., Peurifoy, J., Shen, Y., Tegmark, M., Soljacic, M., Bengio, Y.: Gated orthogonal recurrent units: On learning to forget. Neural computation 31(4), 765-783 (2019)

14. Knoll, J., Groß, R., Schwanke, A., Rinn, B., Schreyer, M.: Applying recommender approaches to the real estate e-commerce market. In: International Conference on Innovations for Community Services. pp. 111-126. Springer (2018)

15. Qadir, H., Khalid, O., Khan, M.U.S., Khan, A.U.R., Nawaz, R.: An optimal ride sharing recommendation framework for carpooling services. IEEE Access 6, 6229662313 (2018). https://doi.org/10.1109/ACCESS.2018.2876595

16. Quadrana, M., Karatzoglou, A., Hidasi, B., Cremonesi, P.: Personalizing sessionbased recommendations with hierarchical recurrent neural networks. In: Proceedings of the Eleventh ACM Conference on Recommender Systems. pp. 130-137. ACM (2017)

17. Ricci, F., Rokach, L., Shapira, B.: Chapter 1 introduction to recommender systems handbook. In: Recommender Systems Handbook (2010)

18. Shearin, S., Lieberman, H.: Intelligent profiling by example. In: Proceedings of the 6 th international conference on Intelligent user interfaces. pp. 145-151. ACM (2001)

19. Tan, Y.K., Xu, X., Liu, Y.: Improved recurrent neural networks for session-based recommendations. In: DLRS@RecSys (2016)

20. Tonara, D.B., Widyawono, A.A., Ciputra, U.: Recommender System in Property Business a Case Study from Surabaya, Indonesia. SPECIAL ISSUE- International Journal of the Computer, the Internet and Management 23(May), 30-31 (2013)

21. Wu, C.Y., Ahmed, A., Beutel, A., Smola, A.J., Jing, H.: Recurrent recommender networks. In: Proceedings of the tenth ACM international conference on web search and data mining. pp. 495-503. ACM (2017)

22. Yuan, X., Lee, J.H., Kim, S.J., Kim, Y.H.: Toward a user-oriented recommendation system for real estate websites. Information Systems 38(2), 231-243 (2013) 